\title{
Lidil
}

Revue de linguistique et de didactique des langues

\section{Acquisition des liaisons prénominales : complémentarité des approches transversale et longitudinale}

Acquisition of Prenominal Liaison: What Can We Learn from the

Complementarity of Cross-Sectional and Longitudinal Approaches?

\section{Céline Dugua et Jean-Pierre Chevrot}

\section{OpenEdition}

Journals

Édition électronique

URL : http://journals.openedition.org/lidil/3695

DOI : 10.4000/lidil.3695

ISSN : 1960-6052

Éditeur

UGA Éditions/Université Grenoble Alpes

Édition imprimée

Date de publication : 30 mai 2015

Pagination : 35-63

ISBN : 978-2-84310-297-4

ISSN : $1146-6480$

\section{Référence électronique}

Céline Dugua et Jean-Pierre Chevrot, « Acquisition des liaisons prénominales : complémentarité des approches transversale et longitudinale », Lidil [En ligne], 51 | 2015, mis en ligne le 30 novembre 2016, consulté le 19 avril 2019. URL : http://journals.openedition.org/lidil/3695; DOI : 10.4000/lidil.3695 


\title{
Acquisition des liaisons prénominales : complémentarité des approches transversale et longitudinale
}

\author{
Céline Dugua* et Jean-Pierre Chevrot**
}

\begin{abstract}
RÉSUMÉ
Le présent article concerne l'acquisition des liaisons prénominales qui apparaissent en français entre les déterminants et les noms. Nous présentons d'abord un modèle constructionniste d'acquisition de ces liaisons qui prend appui sur les principes des théories basées sur l'usage et les grammaires de construction. Cet ancrage théorique nous permet de traiter la liaison comme un phénomène non seulement phonologique, mais aussi comme un moyen d'accéder à des questions plus larges telles que la segmentation des mots nouveaux et la mise en place de la structure de base du groupe nominal. Du point de vue méthodologique, nous défendons l'idée que l'étude de l'acquisition des liaisons a bénéficié du croisement de différentes approches : études de cas fondées sur des corpus, relevé longitudinal des erreurs enfantines, tâches expérimentales sollicitant des jugements ou la production de liaisons ciblées, expériences d'amorçage et chronométrie mentale. Plusieurs de ces méthodes ont été mises en œuvre sur d'importants échantillons d'enfants lors d'études transversales. Dans cet article, nous présentons le suivi longitudinal de 20 enfants entre 2 et 6 ans. Ils participent à quatre tâches explorant différents aspects de la liaison. Les tendances longitudinales sont mises en perspective avec les résultats d'une étude transversale incluant 200 enfants de la même période biographique.
\end{abstract}

\section{ABSTRACT}

This paper is about the acquisition of prenominal liaison, which appears between a determiner and a noun in the French connected speech. First we present a constructionist model of liaison acquisition grounded in the principles of the usage-based theory of language acquisition. This

* Laboratoire ligérien de linguistique, UMR 7270, Université d'Orléans.

** LIDILEM, Université de Grenoble et Institut universitaire de France. 
theoretical viewpoint allows us to consider liaison not only as a phonological phenomenon but also as a window into broader issues such as segmentation of new words and the early construction of the structure of nominal phrase. Our methodological viewpoint is that the study of liaison acquisition has benefited from the complementarity of methods: corpus-based case studies, recording of child errors during everyday interaction, experiments eliciting acceptability judgment or production of targeted liaison, priming experiments, reaction-time experiments. Several of these methods have been implemented to large samples of children in the form of cross-sectional studies. The present study is based on the longitudinal follow-up of twenty children from 2 to 6 years of age. They participated to four tasks exploring different aspects of liaison. The longitudinal trends are compared with the results of a crosssectional study involving 200 children aged from 2 to 6.

\section{Introduction}

Depuis les années 2000, la question de l'acquisition de la liaison a fait l'objet d'études nombreuses, participant à un mouvement plus général de réexamen de ce phénomène de sandhi ${ }^{1}$ (Côté, 2005; Laks, 2005), parmi celles-ci, des études expérimentales qui ont donné lieu à un modèle d'acquisition basé sur l'usage. Le but de cet article est de présenter ce dernier, et de le confronter à de nouvelles données issues d'échantillons d'enfants âgés entre 2 et 6 ans et participant à un suivi longitudinal. Sur le plan factuel, la liaison se caractérise par la production d'une consonne entre deux mots (désormais Mot1 et Mot2), lorsque le Mot1 se termine à l'écrit par une consonne muette et le Mot2 commence par une voyelle; par exemple, dans la suite Mot1-Mot2 un avion /õnavjõ/, apparait une liaison en $/ \mathrm{n} /$. La nature de la consonne produite (consonne de liaison ou CL) dépend du Mot1:/n/ après un, aucun, etc., /z/ après des, les, deux, etc., /t/ après petit, grand, etc. (pour plus de précision, voir Dugua, 2006). La définition des contextes où les liaisons peuvent ou doivent se réaliser ainsi que l'étude des facteurs de leur réalisation font intervenir plusieurs niveaux de fonctionnement du langage : morphosyntaxe, lexique, orthographe, paramètres sociaux et contextuels.

1. Andersen (1986) adopte du sandhi une définition large qui inclut toute réorganisation segmentale ou prosodique, continue ou discontinue, associée à une combinaison de signes linguistiques et qui survient à leur frontière. 
Sur le plan théorique, la liaison pose donc la question de l'autonomie de la phonologie (Chevrot, Fayol \& Laks, 2005 ; Wauquier, 2009).

Nous défendrons ici l'idée que l'acquisition de la liaison ne peut être ni décrite ni comprise si on restreint les faits et les modèles au domaine de l'acquisition phonologique tel que Core $(2012$, p. 80) le circonscrit, à savoir l'acquisition d'un système de phonèmes définis par des traits, la connaissance de la production et de la perception de ces phonèmes, la maitrise des aspects phonotactiques et suprasegmentaux. Du fait de sa position à la frontière entre deux mots, de son caractère variable en fonction du premier mot, de la définition des contextes de réalisation, la liaison implique des relations interactives entre niveaux linguistiques. Pour cette raison, nous proposons un modèle d'acquisition des liaisons prénominales dans le cadre des théories fondées sur l'usage et des grammaires de construction, particulièrement aptes à rendre compte de cette interactivité (pour une discussion entre cette conception et une approche phonologique, voir Chevrot, Dugua, HarnoisDelpiano, Siccardi \& Spinelli, 2013; Wauquier, 2009). Vue à travers ce prisme, l'acquisition de la liaison est donc une fenêtre ouverte sur des phénomènes qui, tout en incluant le niveau phonologique, le dépassent largement. Plus précisément, notre modèle met en relation l'acquisition des liaisons prénominales avec la segmentation des mots nouveaux, la construction des formes lexicales et la mise en place de la structure de base du groupe nominal.

Dans cet article, nous reviendrons tout d'abord sur quelques principes des cadres théoriques dans lesquels s'inscrit ce travail, puis nous présenterons les deux étapes du modèle constructionniste d'acquisition des liaisons prénominales. Enfin, après avoir rappelé les différentes méthodologies mises en place pour élaborer ce modèle, nous rendrons compte de la complémentarité de données résultant de deux types d'observation, l'une transversale, et l'autre longitudinale.

\section{Retour sur quelques principes des approches basées sur l'usage et des grammaires de constructions}

Par leurs principes (Kemmer \& Barlow, 2000 ; Tomasello, 2003), les théories basées sur l'usage constituent une approche adéquate pour rendre compte des interfaces linguistiques et des relations entre formes linguistiques, inscriptions sociales et traitements cognitifs. D'un point de vue développemental, elles s'inscrivent dans les approches constructivistes, dans la lignée des travaux de Piaget (1936), c'est-à-dire dans 
un cadre où les structures mentales abstraites se construisent progressivement, stade après stade, à travers l'interaction entre l'individu et son environnement. C'est à ce niveau qu'intervient la notion même d'usage, à savoir les différentes sources d'input langagier et les interactions à partir desquelles l'enfant construit son système linguistique.

Selon Tomasello (2003), le système linguistique du locuteur est fondé sur les «évènements d'usage », c'est-à-dire les énoncés concrets que le locuteur produit et entend. En ce sens, les représentations linguistiques leur sont étroitement liées puisque les évènements d'usage constituent la base à partir de laquelle le système linguistique du locuteur se forme et évolue. Dans cette perspective, les processus de mémorisation permettent le stockage de séquences concrètes de mots lors du contact avec les évènements d'usage rencontrés dans l'environnement, et les traitements analogiques font le lien entre ces instances concrètes mémorisées et des généralisations plus abstraites. Ainsi, l'abstraction est bien présente dans l'approche basée sur l'usage, mais elle est lente, graduelle et fondée sur des processus plus concrets.

De ce fait, les relations entre les évènements d'usage du locuteur et les représentations abstraites de sa grammaire sont constantes et se réalisent par ce que Kemmer et Barlow (2000) considèrent comme un phénomène d'émergence. Les représentations se construisent par un processus d'analogie qui met en relation des instances spécifiques récupérées dans l'environnement. Ainsi, aucune représentation abstraite ne peut exister sans qu'elle soit ou qu'elle ait été sous-tendue par un contenu concret, autrement dit un contenu phonologique et lexical. Ce processus rend le système linguistique fondamentalement dynamique : à tout moment - et plus particulièrement lors des phases de formation et d'instabilité - des influences interactives agissent pour se diffuser, des représentations phonétiques aux constructions abstraites en passant par les strates intermédiaires, des constructions qui comprennent à la fois des éléments phonétiques et des représentations abstraites.

Les grammaires de construction sont des théories grammaticales dans lesquelles les unités de base du traitement et des représentations linguistiques sont des objets théoriques nommés «constructions » (Croft \& Cruse, 2004; Goldberg, 1995 ; Langacker, 1987). Grammaires de construction et approches basées sur l'usage sont fréquemment rapprochées puisque les premières fournissent l'unité qui permet de lier les évènements d'usage aux représentations linguistiques. Les constructions sont des unités linguistiques à deux faces indissociables l'une de l'autre, à savoir des appariements conventionnels entre formes phonolo- 
giques et fonctions sémantiques ou pragmatiques (Diessel, 2004; Goldberg, 1995, 2003; Langacker, 1987, 2000). Elles peuvent être constituées d'un seul mot ou d'une suite de mots et varient selon une échelle de schématisation et d'abstraction, entre un pôle constitué de constructions concrètes informées phonologiquement - de type expressions idiomatiques (Fillmore, Kaye \& O'connor, 1988) - et un pôle formé de constructions abstraites, appelées aussi schémas. Entre ces deux pôles, s'organise un ensemble de constructions mixtes qui combinent des éléments concrets et des éléments abstraits (Croft \& Cruse, 2004). Ces constructions, de longueur et de niveau d'abstraction différents, coexistent dans le système linguistique du locuteur, comme évoqué plus haut.

Le cadre fourni par les théories basées sur l'usage et les grammaires de construction est celui que nous avons choisi pour rendre compte du développement de la liaison au sein du modèle constructionniste que nous défendons. La construction, telle que définie dans cette approche, constitue pour nous un outil fécond et flexible. En ne spécifiant pas d'emblée le niveau et le degré d'abstraction auxquels l'enfant opère des appariements entre formes et fonctions, la notion de construction rend compte du fait que ni la notion de mot, ni la séparation entre les différents niveaux linguistiques ne sont données aux enfants pré-lecteurs qui doivent extraire l'organisation de leur langue des messages globaux et des situations de communication. Dans notre modèle, nous traiterons donc la liaison non comme un segment phonologique variable pouvant apparaitre entre deux mots, mais comme un élément constitutif d'un ensemble de constructions, qu'il s'agisse de séquences figées concrètes intégrant cette consonne ou de schémas abstraits tenant compte de sa variabilité et des facteurs qui la conditionnent (Bybee, 2003).

\section{Présentation du modèle constructionniste d'acquisition de la liaison prénominale}

Parallèlement au modèle de Tomasello (2003) sur le développement des constructions verbales en anglais, nos précédents travaux (Dugua, 2006; Chevrot, Dugua \& Fayol, 2009), élaborés à partir de nombreuses données empiriques sur lesquelles nous revenons par la suite, nous permettent de proposer un modèle d'acquisition de la liaison catégorique prénominale entre un déterminant et un nom. Ce modèle qui rend compte de la construction du groupe nominal - plus particulièrement de sa structure de base en français : déterminant-nom - repose sur le principe 
d'un lexique enfantin hétérogène, c'est-à-dire composé à la fois de constructions concrètes comprenant plusieurs «mots» adultes, de mots isolés (Pine \& Lieven, 1993, 1997) segmentés à partir de l'input et de constructions plus abstraites. Il constitue en outre une approche alternative au modèle développé par Wauquier (2009; Wauquier-Gravelines \& Braud, 2005) qui considère que l'enfant construit une représentation phonologique abstraite de la liaison en exploitant, dans le cadre d'une grammaire en voie de paramétrisation, sa connaissance des contraintes phonologiques universelles et des régularités morphologiques.

En français, les séquences «déterminant-nom» sont très fréquentes, il est donc attendu que précocement, les enfants mémorisent certaines d'entre elles comme des touts non segmentés. Dans un corpus d'erreurs relevées au vol chez une fillette de 2 ans et demi, Dugua (2006) observe des productions de type un lalampe, un latrompe, qui suggèrent que les séquences «déterminant-nom» la lampe et la trompe ont été mémorisées comme des touts puis introduites dans une structure nominale comprenant un déterminant. La mémorisation globale de groupes nominaux a d'ailleurs été mise en évidence par des expérimentations menées chez des adultes hispanophones (Janssen \& Barber, 2012) ou des études de corpus réalisées auprès d'enfants anglophones de 2-3 ans (Pine, Freudenthal, Krajewski \& Gobet, 2013).

Le contexte de liaison intervenant entre déterminant et nom (ex : des avions, un âne, etc.) est unanimement considéré comme obligatoire (Delattre, 1966; Encrevé, 1983; De Jong, 1994), il n’y a donc pas de variation dans son usage, tous les adultes réalisant cette liaison dans la quasi-totalité de ses occurrences (Durand \& Lyche, 2008). Dans une perspective basée sur l'usage, l'invariance de la liaison obligatoire doit être soulignée puisqu'elle présuppose que les enfants, quel que soit leur environnement langagier, entendent les constructions déterminant-nom avec liaison sous une seule forme.

Le modèle d'acquisition de la liaison catégorique permet de mettre en évidence la façon dont les principes des théories basées sur l'usage s'organisent dans le développement. Il se présente en deux étapes (Nardy \& Dugua, 2011).

\section{1. Étape 1 : segmentation d'exemplaires de noms et construction de schémas généraux}

La liaison prénominale crée un phénomène de resyllabation aboutissant à une dissociation entre les frontières lexicale et syllabique. La séquence les avions est ainsi syllabée /le.zavjõ/, la frontière syllabique se place avant 
le /z/ initial de la syllabe /za/. De plus, la consonne de liaison produite variant en fonction du déterminant, les enfants vont rencontrer chaque nom précédé des différentes consonnes de liaison $-/ \mathrm{n} /$ dans un avion, /z/ dans les avions, /t/ dans petit avion - ou précédé de la consonne provenant de l'élision - /1/ dans l'avion - ou sous une forme sans consonne de liaison comme dans joli avion. Dans cette situation, trois raisons convergentes conduiraient les enfants de 2-4 ans à placer les frontières lexicales avant les différentes consonnes de liaison. Premièrement, les processus précoces de segmentation lexicale exploitent les correspondances probables entre début de mot et attaque syllabique (Goyet, De Schonen \& Nazzi, 2010; Mattys \& Jusczyk, 2001). Deuxièmement, en français les mots commencent approximativement trois fois plus souvent par une consonne que par une voyelle (Chevrot, Dugua \& Fayol, 2009). Troisièmement, aussi bien en allemand qu'en français ou en anglais, les enfants utilisent les déterminants fréquents comme repère pour segmenter le nom suivant (Höhle \& Weissenborn, 2000; Shi, Marquis \& Gauthier, 2006; Shi \& Lepage, 2008). La liaison restant un phénomène minoritaire dans le groupe nominal, il est probable que les enfants mémorisent les déterminants les et un sous les formes /le/ et /õ/ et qu'ils segmentent /lezavion/ (les avions) en plaçant l'initiale du nom après /le/ et en traitant la séquence suivante /zavjõ/ comme une forme possible du nom avion. La forte pression exercée par ces raisons convergentes conduirait, dans certains cas, les jeunes locuteurs à opérer des segmentations privilégiant des débuts de mots de type consonnevoyelle $(\mathrm{CV})$ dans les différents contextes où apparait un nom. Ils seront donc amenés à mémoriser plusieurs variantes lexicales de certains noms :

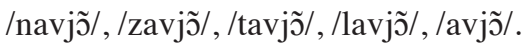

Parallèlement, les séquences mémorisées sont mises en relation les unes avec les autres. Des régularités s'organisent autour du déterminant, qui constitue un élément fréquent, stable et toujours antéposé au nom dans lesdites séquences. Grâce à ce déterminant, des schémas généraux ou schémas pivots pourront émerger. À partir des liens établis entre les

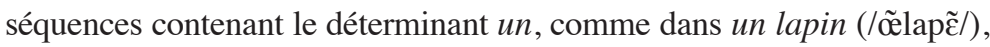

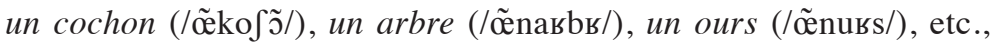
l'enfant construirait un schéma de type $u n+/ \mathrm{X} /$. Dans cette notation, le $/ \mathrm{X} /$ représente un emplacement vide dans lequel peut s'insérer tout type de construction en mémoire. Puisque ces schémas ne contiennent aucune information sur la variante du nom qu'il convient d'insérer après chaque déterminant, c'est lors de cette étape que les enfants produisent des erreurs par remplacement (/õzuss/ pour un ours), des erreurs par 
omission (/deavjã/ pour des avions) et des productions de type /õlalãp/ un lalampe pour une lampe (Dugua, 2006). Ainsi, l'erreur /õzurs/ résulterait de l'insertion de la variante /zuss/ après le déterminant un.

\section{2. Étape 2 : construction de schémas abstraits généralisant la relation entre un déterminant et des exemplaires de noms}

Alors que l'enfant continue d'élaborer des schémas issus de la première étape $(u n+/ \mathrm{X} /$, deux $+/ \mathrm{X} /)$, vers l'âge de 4 ans, il a mémorisé la relation entre certains déterminants et la variante adéquate du nom. Il apprend que l'exemplaire /navjõ/suit le déterminant $u n$, que /zavj̃̃/ suit le déterminant des, etc. Cet apprentissage résulte de l'exposition aux séquences bien formées contenant une liaison rencontrée dans son environnement langagier et de la mémorisation globale de certaines d'entre elles. L'input permettrait ainsi de structurer le réseau d'associations entre des déterminants particuliers et des variantes de noms et de généraliser les liens qui unissent un déterminant particulier à une classe d'exemplaires de noms.

Cette généralisation conduit l'enfant à élaborer des schémas spécifiés de type les $+/ \mathrm{zX} /$ ou $u n+/ \mathrm{nX} /$ qui précisent que l'emplacement qui suit le déterminant les commence par une variante en /z/ initial et l'emplacement qui suit le déterminant un commence par une variante en $/ \mathrm{n} /$ initial. Ces schémas spécifiés permettent alors de produire des liaisons justes sans devoir rencontrer et mémoriser toutes les combinaisons possibles des déterminants et des variantes liaisonnées des noms. Par exemple, un enfant qui n'aurait pas mémorisé l'exemplaire /zuss/, à défaut d'avoir rencontré suffisamment souvent le mot ours précédé de la consonne de liaison $/ \mathrm{z} /$, pourra produire correctement la séquence les ours. À partir des exemplaires du mot ours dont dispose l'enfant dans son lexique (/nuss/, /turs/, /luss/, /uss/), l'exemplaire /zuss/ serait créé par l'assimilation, par le schéma les $+/ \mathrm{zX} /$, de l'un de ces exemplaires disponibles en mémoire.

En outre, ce schéma spécifié mène l'enfant à produire des erreurs dans des séquences déterminant-nom où le déterminant suscite la liaison et le nom commence par une consonne potentiellement consonne de liaison $-/ \mathrm{n} /$ ou $/ \mathrm{z} /$ - comme par exemple zèbre ou nombril. Dans ce type d'erreurs, le /n/ ou le /z/ initial du nom est remplacé par la consonne de liaison compatible avec le déterminant. Par exemple, l'activation du schéma spécifié $u n+/ \mathrm{nX} /$ conduit l'enfant à produire la séquence $u n$

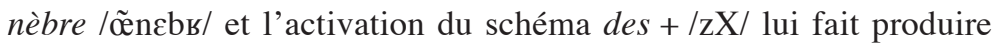


des zombrils /dezõbril/ plutôt que un zèbre /ãzzbь/ ou des nombrils /denõbsil/. Comme l'a montré Dugua (2006), les taux d'erreurs par remplacement de la consonne initiale compatibles avec le déterminant présentent une trajectoire développementale typique des régularisations (Berko, 1958) : leur fréquence est faible jusqu'à 4 ans, elle augmente vers 4-5 ans, puis diminue vers 5-6 ans. En outre, ces erreurs ne peuvent pas être imputées à de fausses segmentations (/zвbь/ segmenté / عbь/ et resyllabé avec une liaison située à la finale de un), les enfants qui effectuent les erreurs /õnєbь/ ou /dezõbьil/ savent parfaitement que zèbre commencent par /z/ et nombril par /n/ (Chevrot, Dugua \& Fayol, 2009). Ces erreurs de régularisation confortent d'une part, l'existence des schémas porteurs de liaison et d'autre part, ils attestent de leur capacité d'assimilation d'un matériau linguistique nouveau, c'est-à-dire de leur productivité.

\section{Aperçu des méthodes pour étudier l'acquisition des liaisons}

Un des principes des théories basées sur l'usage recensés par Kemmer et Barlow (2000) souligne l'importance des données issues de l'usage pour la description et la construction de théories sur le langage (voir Nardy, 2008 pour une synthèse). Ce principe méthodologique s'avère totalement cohérent avec les conceptions théoriques défendues dans cette approche : si le système linguistique est étroitement lié à l'usage, les théories linguistiques doivent se fonder sur l'observation des usages réels des locuteurs et non exclusivement sur des exemples construits ou sur des intuitions. L'étude des productions prises dans leur contexte constitue donc l'approche méthodologique privilégiée.

Historiquement, les premiers recueils d'énoncés enfantins contenant des erreurs de liaison se trouvent recensés dans des journaux de bord, réalisés par des parents observant leurs propres enfants (Grégoire, 1947), mais la question de la liaison n'a jamais fait l'objet d'études spécifiques. Un recueil plus récent a été réalisé par J.-P. Chevrot entre les années 1997 et 2002. Durant 4 ans, il reportait sur des carnets les erreurs de liaison, et plus généralement de segmentation, produites par sa fille alors âgée entre $2 ; 1$ et $6 ; 4$, en notant également l'énoncé porteur de l'erreur. Chevrot et Fayol (2000) ont décrit et analysé 665 de ces erreurs. Le corpus a ensuite été complété pour atteindre un total de 898 erreurs, analysées par Dugua (2006) et Dugua et Spinelli (2009). Ce corpus constitue une source majeure d'informations à la fois sur la 
typologie des formes produites et sur le décours développemental des erreurs.

C'est à partir d'hypothèses résultant de ces données qu'ont été mises en place des tâches expérimentales sollicitant des productions ou des jugements d'acceptabilité, proposées à d'importants échantillons d'enfants lors d'études transversales (Chevrot, Dugua \& Fayol, 2009; Chevrot, Nardy \& Barbu, 2011; Barbu, Nardy, Chevrot \& Juhel, 2013), ainsi que des expérimentations de chronométrie mentale (Dugua, Spinelli, Chevrot \& Fayol, 2009; Gallot, Spinelli, Chevrot \& Dugua, 2009). Ces différentes démarches articulant recueils écologiques et expérimentations ont permis d'établir et de documenter une typologie des erreurs de liaisons. On a ainsi pu mieux comprendre ces productions erronées en les reliant à des questions générales de segmentation et de construction du groupe nominal, stabiliser des repères développementaux et tester certaines prédictions du modèle. Plus récemment, de nouvelles démarches ont commencé à examiner les liaisons dans le discours adressé à l'enfant dans des corpus longitudinaux d'interactions familiales (Liégeois, Chabanal \& Chanier, 2011). Sur le versant expérimental, les étapes précoces du modèle sont actuellement testées à travers des tâches impliquant la technique du «regard préférentiel»ou la segmentation de pseudo-noms, proposées à des enfants de moins de 3 ans (Babineau \& Shi, 2014; Buerkin-Salgado, Culbertson, Legendre $\&$ Nazzi, 2014).

En bref, notre modèle développemental de l'acquisition de la liaison présenté précédemment résulte d'observations écologiques à partir desquelles ont été élaborés plusieurs protocoles expérimentaux qui ont permis de tester les hypothèses initiales et de déterminer des repères développementaux jalonnant le décours temporel des erreurs et des formes justes. Jusqu'à ce jour, les débats ont donc bénéficié du croisement des différentes méthodologies et des va-et-vient nécessaires entre elles, un éclectisme méthodologique que soutiennent d'ailleurs les tenants des théories basées sur l'usage (Kemmer \& Barlow, 2000; Tomasello, 2003).

Néanmoins, les travaux sur la liaison ne dérogent pas à une division des approches méthodologiques courante dans le domaine de l'acquisition du langage oral, les études longitudinales concernant plus généralement des travaux sur corpus, alors que les études transversales impliquent des tâches expérimentales. Cette répartition introduit une difficulté. En effet, les expérimentations présentent l'intérêt d'être conçues pour tester des hypothèses précises prédites par les modèles. 
Toutefois, leur mise en œuvre transversale interdit d'examiner les trajectoires développementales des mêmes individus, puisqu'elles comparent des groupes d'âge composés d'individus différents. En outre, le raisonnement statistique à partir de moyennes de groupes d'âge risque de minimiser la variabilité interindividuelle. L'approche transversale est donc susceptible de favoriser la généralisation de modèles qui ne concerneraient en fait qu'une partie des enfants. Dans le but de mieux cerner cette variabilité potentielle des individus et des trajectoires individuelles, nous avons mis en place un suivi longitudinal basé sur quatre tâches expérimentales, identiques à celles proposées lors d'une observation transversale à grande échelle, afin de pouvoir comparer les résultats issus de ces deux modalités d'observation.

\section{Résultats et analyses des approches transversale et longitudinale}

Nous présenterons ici des données obtenues par le biais d'un suivi longitudinal, en les confrontant avec celles résultant du même protocole appliqué lors d'une étude transversale à grande échelle (Dugua, 2006; Chevrot, Dugua \& Fayol, 2009). Cette section est organisée autour des deux grandes étapes du modèle développemental.

\subsection{Sujets}

L'étude longitudinale mise en place consiste en un suivi de 20 enfants (10 filles et 10 garçons), rencontrés à cinq reprises (nous parlerons de cinq temps d'observations) entre les âges de 2;5 et 6;3 et dont les rythmes de recueils sont précisés dans le tableau 1.

\begin{tabular}{ccccc}
\hline $\begin{array}{c}\text { Temps } \\
\text { d'observation }\end{array}$ & $\begin{array}{c}\text { Échelle } \\
\text { d'âge }\end{array}$ & Moyenne & $\begin{array}{c}\text { Déviation } \\
\text { standard }\end{array}$ & $\begin{array}{c}\text { Intervalle de temps } \\
\text { entre t-1 et t }\end{array}$ \\
Temps 1 & $2 ; 5-3 ; 1$ & $2 ; 9$ & 2.3 & 14 mois \\
Temps 2 & $3 ; 7-4 ; 3$ & $3 ; 11$ & 2.2 & 7 mois \\
Temps 3 & $4 ; 2-4 ; 9$ & $4 ; 6$ & 2.1 & 8 mois \\
Temps 4 & $4 ; 9-5 ; 5$ & $5 ; 2$ & 2.3 & 7 mois \\
Temps 5 & $5 ; 6-6 ; 3$ & $5 ; 9$ & 2.3 & \\
\hline
\end{tabular}

Tableau 1. - Échantillon d'enfants de l'étude longitudinale. 
L'étude transversale qui nous sert de point de repère comprend 200 enfants, âgés de 2;4 à 6;1 et répartis en 4 tranches d'âge (tableau 2).

\begin{tabular}{ccccc}
\hline $\begin{array}{c}\text { Tranche } \\
\text { d'âge }\end{array}$ & $\begin{array}{c}\text { Échelle } \\
\text { d'âge }\end{array}$ & Moyenne & $\begin{array}{c}\text { Déviation } \\
\text { standard }\end{array}$ & Effectif \\
Tranche d'âge 1 & $2 ; 4-3 ; 1$ & $2 ; 9$ & 2.3 & 48 enfants \\
Tranche d'âge 2 & $3 ; 2-4 ; 1$ & $3 ; 6$ & 3.5 & 50 enfants \\
Tranche d'âge 3 & $4 ; 2-5 ; 0$ & $4 ; 7$ & 3.1 & 52 enfants \\
Tranche d'âge 4 & $5 ; 2-6 ; 1$ & $5 ; 7$ & 3.4 & 50 enfants \\
\hline
\end{tabular}

Tableau 2. - Échantillon d'enfants de l'étude transversale.

\subsection{Protocole}

Le protocole expérimental des deux études est identique, il comporte quatre tâches de production que nous présenterons succinctement en préambule de chaque analyse (pour une présentation détaillée des tâches, voir Dugua, 2006).

\subsection{Vue d'ensemble des productions de liaisons}

\subsubsection{Tâche de dénomination d'images}

À partir d'une tâche de dénomination d'images en contexte déterminant-nom (quatre noms impliqués : arbre, ours, écureuil, éléphant précédés des déterminants un et deux), nous obtenons le décours développemental des formes justes et des erreurs dans un contexte considéré comme obligatoire.

Les résultats de l'étude transversale (fig. 1), obtenus à partir de moyennes par groupes d'âges ${ }^{2}$, suggèrent une forte augmentation des productions justes jusqu'à 4 ans, puis un palier atteint aux alentours de $80 \%$. Les erreurs par remplacement diminuent régulièrement pour atteindre un seuil marginal à 3,7\% dans la dernière tranche d'âge. En revanche, les erreurs par omission sont relativement stables, fluctuant entre $12 \%$ et $20 \%$ des productions.

2. Le format adopté ici ne nous permet pas de fournir les données complètes (notamment les écarts-types). Celles-ci peuvent être obtenues sur simple demande auprès des auteurs. 
L'observation des résultats dans notre étude longitudinale (fig. 1) valide l'ensemble de ces tendances. Soulignons toutefois que la forte augmentation des productions justes entre 2 et 4 ans observée en transversal (44 points, de $36 \%$ à $80 \%$ ) apparait moindre en longitudinal (30 points), où le taux de productions justes est plus élevé dès le premier temps d'observation. En lien avec cette différence, le taux d'erreurs par remplacement au premier temps d'observation est légèrement supérieur aux productions justes en transversal et inférieur en longitudinal. Remarquons enfin que la persistance des erreurs par omission est avérée dans les deux types d'observation.
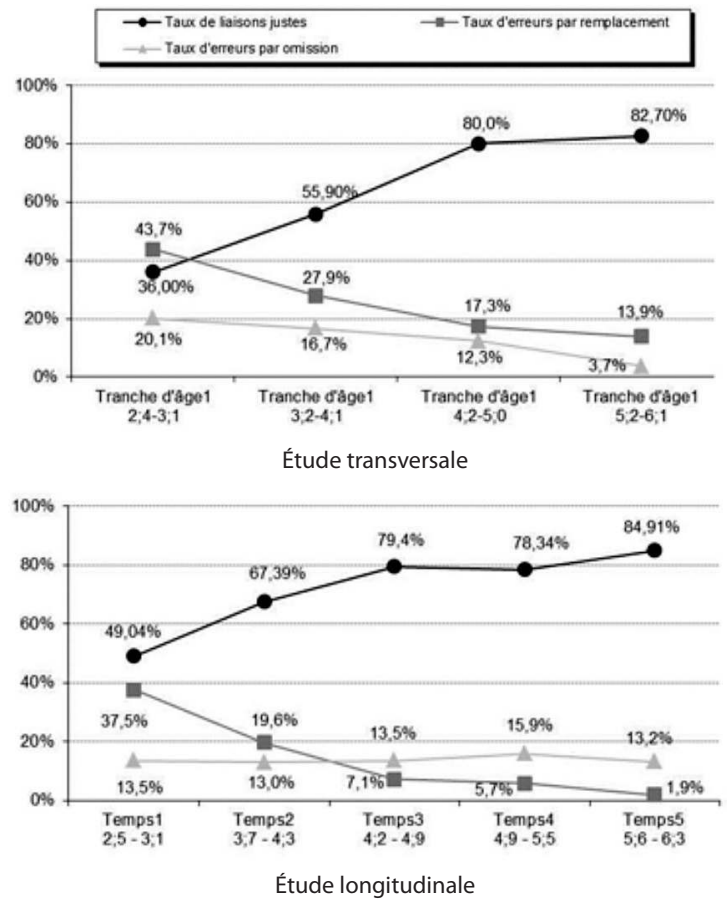

Figure 1. - Résultats obtenus dans la tâche de dénomination entre déterminant et nom.

\section{4. Étape 1 : disponibilité de différents exemplaires de noms}

La première étape de notre modèle se caractérise par la diversité et la disponibilité des variantes lexicales en mémoire. Pour plusieurs noms rencontrés dans l'environnement, l'enfant favoriserait les segmentations à consonne initiale et récupèrerait des variantes commençant par 
des consonnes différentes. La tâche d'apostrophe constitue une façon détournée d'accéder à certaines formes disponibles dans le lexique de l'enfant.

\subsubsection{Tâche d'apostrophe}

Dans cette tâche, les enfants étaient invités à appeler des figurines d'animaux présentées face à eux. La consigne était d'appeler les animaux par leur nom; l'expérimentateur donnait un exemple avec une figurine dont le nom commence par une consonne lapin, lapin, viens ici! L'objectif était d'inciter les enfants à utiliser une structure proche de l'interpellation par des prénoms, donc des structures sans déterminant. Par exemple, pour appeler la figurine de l'ours, produire ours, viens ici! Quatre figurines étaient utilisées, pour faire produire les noms : âne, ours, écureuil, éléphant. Nous cherchions, par cette tâche, à mettre en évidence la présence de consonnes de liaison à l'initiale de variantes lexicales des noms (essentiellement /n/, /z/, /t/ ou le /l/ issu de l'élision).

Trois formes lexicales ont été repérées dans les énoncés produits par les enfants de l'étude transversale : des formes à initiale vocalique (ours, viens ici!), des formes à initiale consonantique (essentiellement en /n/ initial, plus marginalement en /z/ ou en /t/ initial, nours, viens $i c i$ !) et des formes plus longues, impliquant un déterminant et un nom (un + nom et la forme en /1/ initial, l'ours, viens ici!). Entre les âges de 2 et 4 ans, ces trois formes apparaissent dans des proportions comparables. Au-delà de 4 ans, les formes à initiale vocalique deviennent majoritaires, les productions à initiale consonantique diminuent régulièrement, et celles avec déterminant restent relativement stables ${ }^{3}$. En nous attachant aux résultats par Mots2, on peut remarquer que pour les quatre noms, les taux de productions avec déterminant sont comparables. En revanche, on observe des différences sur les deux autres types de productions. Les productions des mots écureuil et éléphant (deux noms longs) sont très majoritairement à voyelle initiale (respectivement $74 \%$ et $69 \%$ des productions), les productions à consonne initiale ne représentant que $7 \%$ et $3,6 \%$. Alors que pour les mots âne et ours (deux noms courts), les productions à voyelle initiale représentent environ $50 \%$, et celles à consonne initiale respectivement $33 \%$ et $25 \%$.

3. Soulignons ici que ces dernières sont majoritairement constituées de formes à initiale en $/ 1 /$, résultant de l'élision du déterminant le. 
Les données longitudinales montrent globalement les mêmes types de résultats : une diversité des productions possibles avant 4 ans, en revanche, un report de quelques mois dans la différenciation des formes à initiale vocalique (après $4 ; 1$ en transversal, après 4;9 en longitudinal). Les deux autres formes suivent des trajectoires comparables à celles obtenues en transversal. Subsistent donc jusqu'à l'âge de 5-6 ans, et quelle que soit la méthodologie adoptée, des productions en /1/ initial, productions qui correspondent à des formes de groupe nominal avec article défini élidé, soit que la non-segmentation du groupe perdure, soit que l'enfant ne maitrise pas les contextes dans lesquels l'article doit être omis.
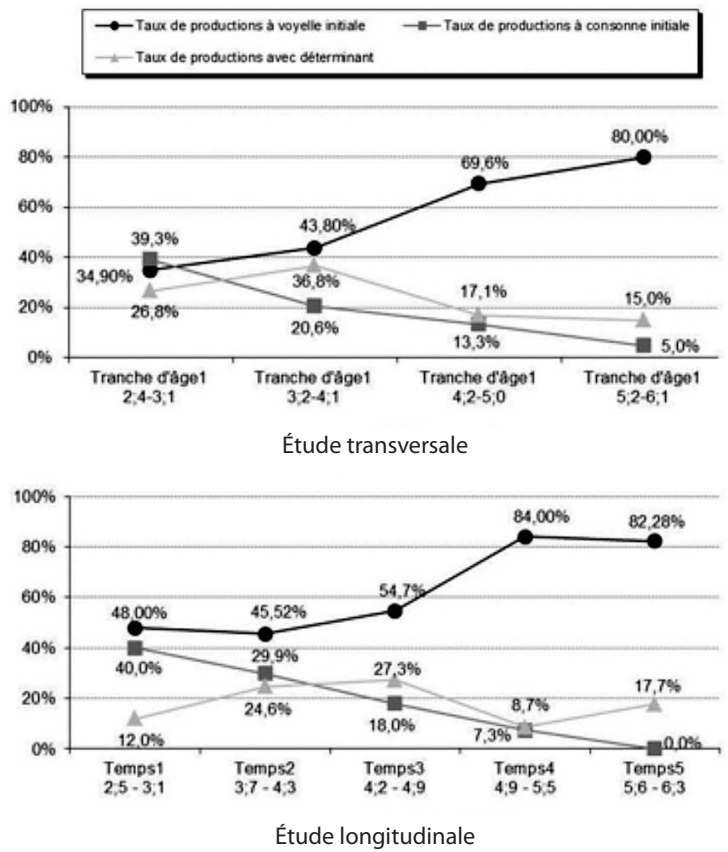

Figure 2. - Résultats obtenus dans la tâche d'apostrophe.

\section{5. Étape 2 : construction de schémas abstraits}

La construction de schémas abstraits garantira à l'enfant de produire correctement les contextes de liaison, étant donné que ces derniers fournissent la forme des noms à produire en fonction du déterminant (les + variantes commençant par $/ \mathrm{z} /$, un + variantes commençant $\mathrm{par} / \mathrm{n} /$, etc.). Nous avons pu observer la productivité de ce type de schémas à travers 
deux tâches expérimentales, dont nous allons comparer les résultats dans leur version transversale et leur version longitudinale.

\subsubsection{Tâche de production de pseudo-noms}

Nous avons créé une tâche de production à partir de quatre pseudonoms (ivak, utrel, ikat, ussa) - représentant chacun un animal imaginaire - entendus par les enfants après les déterminants un et deux. L'expérimentateur désignait par exemple un animal en disant «Là, il y a un $n$-ivak», puis montrait à l'enfant ce même animal en deux exemplaires. À partir des productions des enfants, nous cherchons à mettre en évidence le traitement de la liaison lors de la rencontre avec des mots nouveaux (après l'audition de la séquence $u n$ - $n$-ivak l'enfant produitil deux-z-ivak ou deux-nivak?). Chaque enfant produisait 4 séquences (voir Dugua, 2006 pour plus de précision). Vu ce faible nombre d'occurrences et pour cette tâche uniquement, nous avons réduit les échantillons d'enfants en enlevant ceux qui produisaient plus d'une non-réponse. En transversal, les données s'appuient alors sur 181 enfants, et en longitudinal, sur 16 enfants (4 enfants concernés au premier temps ont été enlevés aux quatre temps suivants).

Les productions s'organisent en trois types :

- les productions dans lesquelles il y a maintien de la forme entendue dans la forme produite : par exemple /ãnivak/ entendu, /dønivak/ produit, /døzysa/ entendu, /õzysa/ produit;

- les productions pour lesquelles l'enfant fait varier la forme du pseudo-nom en adéquation avec le déterminant : p. ex. /ãnivak/ entendu, /døzivak/ produit, /døzysa/ entendu, /œ̃nysa/ produit. Seules les productions de ce type manifestent une maitrise du système de la liaison et de son lien avec le déterminant (/n/ après un, /z/ après deux);

- les productions dans lesquelles aucune consonne n'est produite entre le déterminant et le pseudo-nom, productions comparables aux erreurs dites d'omission décrites dans la tâche de dénomination en contexte de liaison obligatoire : p. ex. /ãnivak/ entendu, /døivak/ produit, /døzysa/ entendu, /õysa/ produit.

On retrouve ces trois types de productions dans l'étude longitudinale, comme dans l'étude transversale, avec des répartitions semblables entre 2 et 4 ans. Au-delà, on repère un décalage dans le temps du moment où se croisent les productions de type maintien et alternance : les productions alternantes deviennent majoritaires plus précocement 
en longitudinal (entre 3 et 4 ans) qu'en transversal (entre 4 et 5 ans). Au-delà de ces points où les formes manifestant la maitrise de l'alternance deviennent majoritaires, on constate que les deux types de productions restent présents. Même si les productions alternantes sont supérieures numériquement aux productions de type maintien, ces dernières ne semblent pas prendre une trajectoire descendante forte qui pourrait être le signe de leur disparition. Remarquons ici que dans le dernier temps d'observation, on relève que $15 \%$ des productions correspondent à des cas où les enfants ont produit les pseudo-noms au

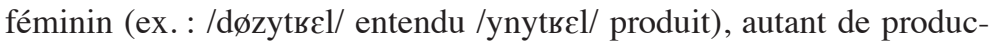
tions qui n'ont pas pu entrer dans les calculs. Enfin, les évolutions des productions avec omission de la consonne sont comparables entre les deux approches : elles se maintiennent globalement dans des moyennes comprises entre $4 \%$ et $16 \%$ des productions.
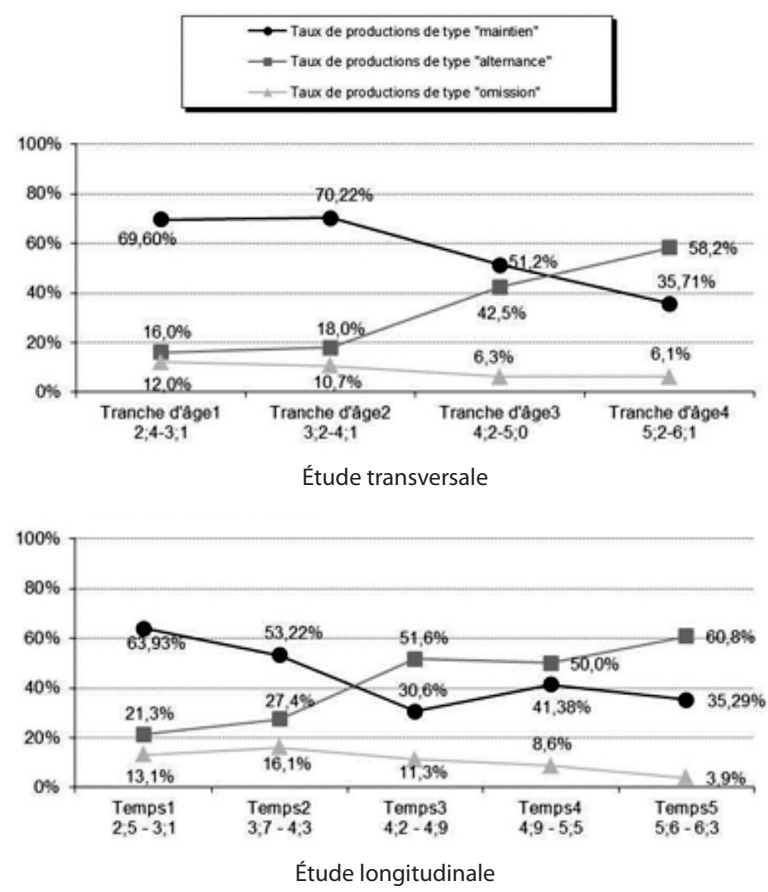

Figure 3. - Résultats de la tâche de production de pseudo-noms après déterminant. 


\subsubsection{Tâche de dénomination de noms à initiale consonantique}

Une tâche de dénomination de noms à initiale consonantique entre les déterminants un et deux et les noms zèbre, nombril, nuage, lavabo a été proposée dans le but de décrire le décours développemental des erreurs de surgénéralisation (un nèbre* pour un zèbre) recueillies ponctuellement dans des corpus écologiques.

La comparaison entre les versions transversales et longitudinales de la tâche fait apparaitre que le taux de productions justes (un/deux zèbre(s), un/deux nuage(s), etc.) est élevé (90\% des productions en longitudinal, $88,9 \%$ en transversal). Outre les productions justes, quatre types d'erreurs ont été relevés dans cette tâche (présentés ici par ordre décroissant de fréquence) :

- des erreurs par remplacement dans lesquelles la consonne à l'initiale du nom est compatible avec le déterminant, comme s'il s'agissait d'une liaison. Ce sont ces erreurs que nous attendons pour la validation de notre modèle. Par exemple, dans /døzyaz/ (deux zuages), la forme /zyaz/ en /z/ initial est semblable aux formes que l'on peut attendre en contexte de liaison après le déterminant deux;

- des erreurs par remplacement dans lesquelles la consonne initiale du nom ne pourrait pas être une liaison compatible avec la nature du déterminant. Par exemple, lorsque l'enfant dit /õzyaz/ (un zuage), la forme /zyaz/ en /z/ initial n'est pas celle qu'on attendrait après le déterminant $u n$;

- des erreurs par remplacement impliquant la consonne /1/, par exemple : /dølyaz/ (deux luages);

- des erreurs par omission de la consonne initiale, par exemple : /døavabo/ (deux avabos).

Le principal résultat est que l'on retrouve, en longitudinal, le pic d'erreurs compatibles avec le déterminant, caractéristique des régularisations prédites par les schémas abstraits de l'étape 2 du modèle constructionniste. En effet, le taux d'erreurs compatibles augmente de 4,17\% au temps 2 à $6,78 \%$ au temps 3 , pour diminuer par la suite. Néanmoins, au premier temps d'observation, nous constatons un taux apparemment important d'erreurs compatibles. Ce taux correspond en fait à trois productions observées chez trois enfants différents, qui constituent d'ailleurs les seules erreurs relevées. En revanche, l'augmentation des erreurs compatibles qui culmine au temps 3 concerne davantage d'enfants : cinq 
enfants voient leurs taux augmenter entre les temps 2 et 3, ces mêmes cinq enfants auxquels s'ajoute un sixième ont des taux qui diminuent entre les temps 3 et 5 .

Par ailleurs, à la différence des résultats en transversal, on observe en longitudinal un autre pic d'erreurs sur les omissions qui étaient des productions relativement importantes et constantes en transversal mais pour lesquelles on n'observait pas d'évolution en pic. Il est donc possible que les omissions manifestent elles aussi un processus de régularisation par lequel l'enfant déduirait les formes à initiale vocalique (ours!) de la connaissance des variantes consonantiques (/zurs/,/nurs/, /turs/).

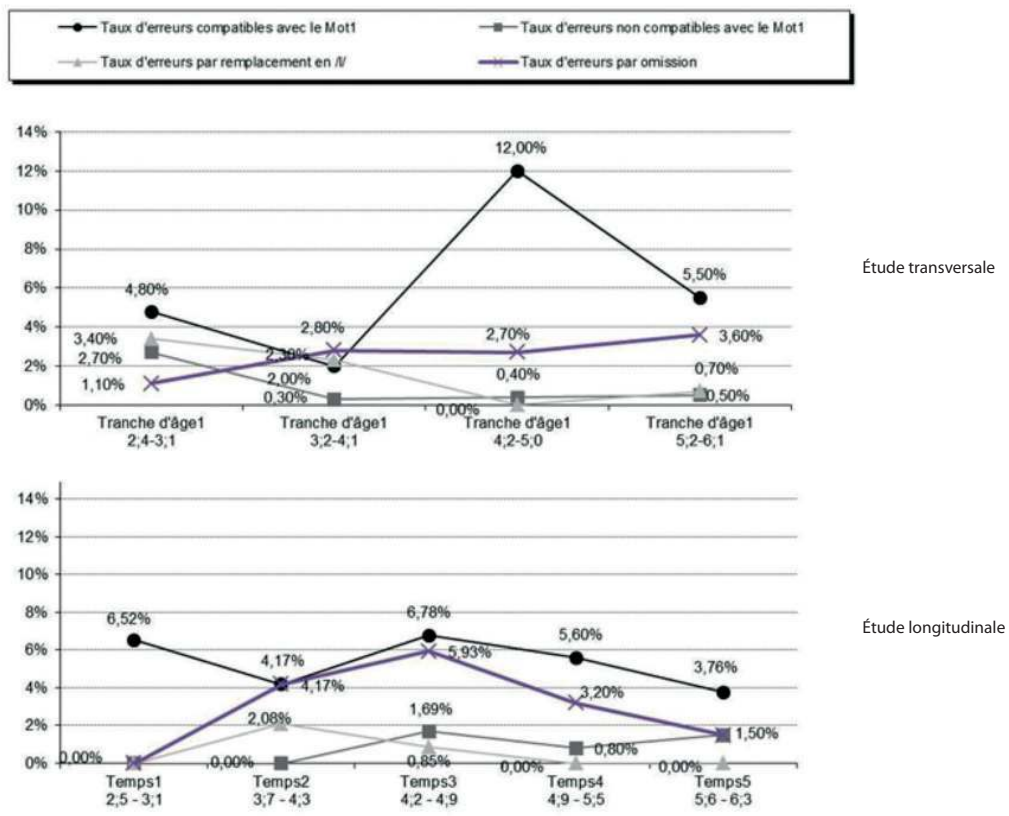

Figure 4. - Résultats de la tâche de dénomination de noms à initiale consonantique après déterminant.

\subsubsection{Analyse inter-tâche dans l'étude longitudinale}

Une étude longitudinale, telle que nous la proposons, permet enfin de comparer les productions de mêmes enfants d'une tâche à l'autre et dans des temps d'observation successifs. Une des questions du modèle porte sur les formes lexicales en mémoire, et leur intégration dans 
des schémas productifs. La variante à initiale vocalique (variante canonique du mot: /ивs/ pour ours, /авbь/ pour arbre, etc.) nous intéresse particulièrement. Est-elle disponible chez les enfants? Est-ce celle qui apparait dans les erreurs par omission? Est-elle nécessaire à la mise en place des liaisons?

Pour tenter d'apporter des éléments de réponses, nous avons établi deux groupes d'enfants à partir des productions obtenues au premier temps d'observation, dans la tâche d'apostrophe, celle justement qui permet d'accéder aux formes lexicales en mémoire :

- Groupe 1 : les enfants qui n'ont produit aucune ou une seule variante à initiale vocalique (sur 8 productions possibles), soit 8 enfants. Dans ce groupe, la proportion de ces variantes par rapport à l'ensemble est de 14,29\%.

- Groupe 2 : les enfants qui ont réalisé au moins deux variantes à initiale vocalique, soit 12 enfants. Les variantes à initiale vocalique représentent pour ce groupe $61,11 \%$ des productions.

À partir de ces deux groupes, nous observons les taux d'erreurs par omission en contexte de liaison obligatoire et ceux de liaisons justes (fig. 5).

Nous constatons sur le graphe du dessus que les enfants du groupe 2 sont ceux qui font le plus d'erreurs par omission, dès le temps 1 et tout au long des recueils, avec une moyenne de $19 \%$. En opposition, les enfants du groupe 1 ne font que peu d'erreurs par omission jusqu'à 4;6 environ, elles émergent par la suite.

Quant aux productions justes, globalement, on n'observe pas de différence entre les deux groupes sur l'ensemble de la période observée, les scores passant globalement de $50 \%$ au premier temps d'observation à $85 \%$ au dernier. Toutefois, l'évolution est plus régulière pour les enfants du groupe 2 , qui produisent plus souvent des variantes à initiale vocalique lors de la tâche d'apostrophe. Les enfants du groupe 1, qui produisent moins souvent des variantes à initiale vocalique lors de la tâche d'apostrophe, manifestent une augmentation plus forte entre les deux premiers temps (environ 30 points), puis une stabilisation entre $80 \%$ et $89 \%$. En bref, les enfants qui ne disposent pas de la variante à initiale vocalique semblent progresser plus précocement dans les productions de liaisons obligatoires, alors que ceux qui en disposent réalisent davantage d'erreurs par omission. La connaissance de la variante à initiale vocalique n'apparait donc pas comme une condition sine qua non de l'apprentissage de la liaison. 


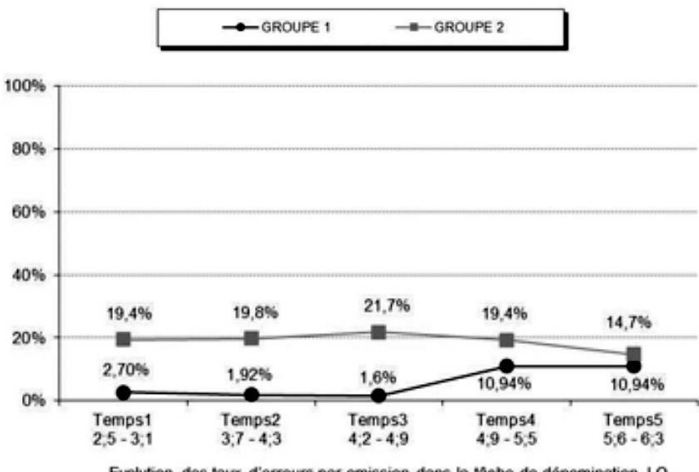

Evolution des tatx d'erreurs par cenission dans la tache de déncmination LO

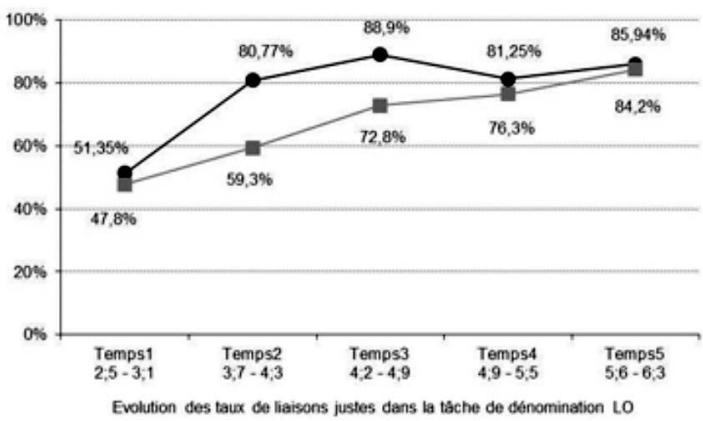

Figure 5. - Résultats de la tâche de dénomination entre déterminant et nom en fonction de la disponibilité de la variante à initiale vocalique.

\section{Recueil transversal et recueil longitudinal : quelles convergences?}

La possibilité de mettre en parallèle un même protocole de production mené dans deux modalités de recueil - recueil transversal et recueil longitudinal - permet d'étayer les modèles théoriques tout en évaluant d'éventuels impacts méthodologiques.

Nous constatons que globalement, les données obtenues à partir d'un suivi longitudinal confirment celles recueillies en transversal qui sont à l'origine du modèle constructionniste d'acquisition de la liaison. L'évolution des taux de liaisons justes ainsi que les types d'erreurs et leurs décours suivent des trajectoires semblables, à ceci près que les enfants de l'échantillon suivi en longitudinal sont globalement plus précoces en ce qui concerne la liaison que ceux de l'étude transversale. En longitudinal, on retrouve les trois mêmes types de variantes rendues accessibles 
par la tâche d'apostrophe : les variantes à voyelle initiale, à consonne initiale et celles précédées d'un déterminant, avec des évolutions comparables aux résultats obtenus en transversal. S'agissant des stratégies de segmentation repérées par une tâche de production de pseudo-noms, elles présentent une évolution proche, la préférence pour les segmentations de type liaison (segmentations dites alternantes) étant un peu plus précoce pour le groupe observé en longitudinal, résultat qui est certainement en lien avec les meilleures performances en liaison pour cet échantillon. Enfin, le pic d'erreurs par surgénéralisation (sur les mots à consonne initiale) est repéré en longitudinal (/døzyaz/), accompagné d'un pic d'erreurs sur des productions sans consonne de liaison (/døyaz/) qu'on ne retrouve pas en transversal. Ces résultats généraux que l'on retrouve dans des suivis longitudinaux confortent donc notre modèle qui, jusque-là, avait été établi à partir de quelques études de cas (Chevrot, Chabanal \& Dugua, 2007) et de statistiques générales, mais qui n'avait jamais été mis à l'épreuve de l'observation suivie des mêmes enfants durant plusieurs années.

Outre ces comparaisons strictes entre les deux approches, nous avons constaté certains résultats qui ne peuvent être obtenus qu'à partir de suivis longitudinaux. Nous avons pu notamment apporter un éclairage nouveau sur le rôle des variantes à voyelle initiale dans le développement de la liaison. Ces dernières sont mémorisées précocement et elles semblent être celles qu'on retrouve plus tard dans les erreurs par omission, erreurs qui résulteraient donc de l'application de schémas généraux dans lesquels l'enfant insère une variante de ce type (des + / avjõ/ aboutissant à /deavjõ/). En revanche, la segmentation précoce de variantes à initiale vocalique ne semble pas être une condition nécessaire à la production des liaisons justes puisque les enfants qui ne disposent pas de cette variante vers 2-3 ans ont des résultats comparables à ceux qui en disposent, et qu'ils progressent même plus vite dans l'usage de la liaison correcte.

Malgré les tendances convergentes entre approche transversale et approche longitudinale, et malgré les apports du suivi longitudinal, certains aspects du modèle contructionniste d'acquisition des liaisons demeurent discutables à la lumière de données nouvelles obtenues chez des enfants plus jeunes. Dans un corpus de trois enfants âgés de 3 ans, Buerkin-Salgado, Culbertson, Legendre \& Nazzi (2014) relèvent que ces derniers ont un taux de réalisation de la liaison de $80 \%$, soit un pourcentage bien supérieur à celui que l'on trouve dans nos tâches de dénomination (en transversal par exemple, dans un sous-échantillon 
reprenant les 20 enfants âgés entre 2;11 et 3;1, les taux de liaisons justes sont en moyenne de $40 \%$, et ils sont de $42 \%$ chez les 9 enfants de l'étude longitudinale dans la même tranche d'âge). De tels écarts manifestent incontestablement un effet des tâches expérimentales de productions - telles que les dénominations utilisées dans nos protocoles - qui sont sources d'erreurs, comparativement à des situations écologiques où les enfants n'ont pas une telle densité de liaisons à produire (Chevrot, Chabanal \& Dugua, 2007). À partir d'une tâche de productions de pseudo-noms après familiarisation dans différents contextes, BuerkinSalgado, Culbertson, Legendre \& Nazzi (2014) suggèrent également que les enfants ont commencé dès 3 ans à développer une représentation de la liaison qui tend davantage vers le modèle adulte que ne le laissent supposer les schémas généraux du modèle constructionniste, qui ne contiennent aucune information sur la liaison. Ensemble, ces deux résultats soulignent que l'usage de la liaison chez les enfants serait plus précoce que ne le prédit ce modèle.

Remarquons tout d'abord que notre modèle est véritablement constructionniste. Puisque chaque enfant construit une représentation des liaisons en fonction du matériau linguistique qu'il rencontre et de ses capacités de traitement, les âges auxquels les jeunes locuteurs franchissent les étapes ainsi que le temps passé dans chacune d'elles sont sujets à des variations interindividuelles. En revanche, l'ordre d'enchainement des stades demeure stable. Finalement, un modèle développemental - quel que soit le cadre théorique où il se développe - fait apparaitre des grandes tendances, et tend ainsi à effacer les trajectoires individuelles.

Un examen plus précis de notre échantillon transversal fait toutefois apparaitre que trois enfants dès 2;6 réalisent $100 \%$ de liaisons justes, et que dix enfants à 4 ans ont un taux de réalisation correcte inférieur à $20 \%$. La variabilité interindividuelle qui est une caractéristique bien repérée de l'acquisition du langage est donc évidemment présente dans la mise en place de la liaison.

D'autres types d'études, menées par Babineau et Shi $(2011,2013)$ chez des enfants encore plus jeunes (20-30 mois) à partir de la technique du regard préférentiel ont mis en évidence leur capacité à discriminer précocement des pseudo-noms à initiale vocalique (dès 24 mois) après qu'ils ont été familiarisés à des séquences impliquant ces formes insérées dans des contextes de liaison (par exemple, discriminer èque, après l'avoir entendu dans mon èque, petit èque, ces èque, dernier èque). Ils concluent : "This result suggests that by 24 months of age infants have 
learned to counter the syllable-alignment bias with subsyllabic statistical segmentation, and this ability led to a vowel-initial interpretation corresponding to the French liaison rule.» Autrement dit, les auteurs établissent un lien entre le fait de segmenter des variantes à voyelle initiale et le fait de maitriser le fonctionnement de la liaison. Ce lien doit être discuté. Dans les protocoles proposés par Babineau et Shi (2011), les formes à initiale vocalique reconnues par les enfants constituent simplement la partie commune des formes auxquelles ils ont été familiarisés dans la première phase du test (la forme èque, constitue la partie commune des formes entendues mon èque, petit èque, ces èque, dernier èque). Ces deux habiletés : segmenter une partie commune et comprendre le fonctionnement de la liaison doivent, selon nous, être distinguées. La première reste une opération générale de segmentation du lexique qui permet, dans ce cas précis, de détacher la consonne de liaison du nom : la forme commune est èque. Mais au-delà de cette capacité, comprendre le fonctionnement de la liaison prénominale nécessite par exemple de savoir associer un déterminant donné à une consonne de liaison particulière, ce dont rendent compte les schémas spécifiés du modèle constructionniste. Babineau et Shi (2013) trouvent justement, par la technique du regard préférentiel, que les enfants de 30 mois ne semblent pas capables de relier ce qu'ils nomment le morphème indépendant $/ \mathrm{z} /$ avec les déterminants correspondant.

Par ailleurs, dans des protocoles tels que les procédures de familiarisation utilisées par Babineau et Shi, l'enfant est confronté à différentes formes d'un même pseudo-nom précédé de quatre consonnes de liaison différentes dans un laps de temps très court. Il reste à se demander si ce type de familiarisation est similaire aux conditions écologiques de découverte des mots en contexte de liaison. Un enjeu actuel est donc d'examiner les liaisons dans le discours adressé aux enfants (voir projet ALIPE en cours, Liégeois, Chabanal \& Chanier, 2011). Afin de contribuer à ce débat, nous avons étudié le corpus Colaje ${ }^{4}$ (Morgenstern \& Parisse, 2012) qui consiste en des enregistrements vidéo d'enfants dans leur milieu familial, à raison d'un enregistrement d'environ une heure par mois. Nous avons pu ainsi accéder à une partie des liaisons que les enfants entendent dans leur environnement. Chevrot (2013) a étudié les groupes nominaux inclus dans les énoncés produits par cinq mères à leurs cinq enfants ( 2 filles et 3 garçons). Les familles sont issues de

4. <http://childes.psy.cmu.edu/browser/index.php?url=Romance/French/Paris/>. 
catégories socioprofessionnelles hautes et l'étude se limite aux deuxième et troisième années des enfants. D'un point de vue quantitatif, ce sous-corpus comprend 18094 groupes nominaux de type déterminantnom produits par les mères dans des énoncés adressés à leur enfant. Dans ces groupes nominaux, seuls $13,3 \%$ des noms sont à initiale vocalique (2408 occurrences) dont 4,9\% sont intégrés dans un contexte de liaison (890 occurrences) et 6,6\% (1204 occurrences) sont précédés du déterminant le élidé. La liaison prénominale est donc un phénomène rare dans le discours adressé à l'enfant, si on le met en perspective avec la fréquence 6 à 7 fois plus élevée des groupes nominaux comprenant un nom à initiale consonantique. L'enfant rencontre donc le plus souvent des mots avec une consonne initiale stable, une caractéristique qui tend à expliquer la longueur du processus d'apprentissage de l'alternance qui caractérise la liaison. D'autre part, les 2094 occurrences de groupes nominaux incluant une liaison ou une élision sont réparties sur 230 noms différents (types). Parmi ces noms, il n'y en a que 5 qui, dans le corpus, apparaissent simultanément derrière les 3 consonnes de liaison et celle de l'élision (/z/, /n/, /t/, /1/). En outre, 150 noms n'apparaissent qu'après une seule de ces quatre consonnes. Autrement dit, dans l'input des enfants, il semble que tous les noms commençant par une voyelle ne sont pas généralement rencontrés derrière l'ensemble des consonnes de liaison et d'élision qui peuvent s'enchainer à leur initiale. Cette restriction résulte de limitations dans les probabilités de combinaisons entre déterminants et noms (par exemple, certains noms appellent un déterminant pluriel du fait de leur sens, Dugua, Spinelli, Chevrot \& Fayol, 2009). Même si ces résultats méritent d'être affinés, puisqu'ils ne reposent pas sur un corpus dense échantillonnant le discours maternel de façon suffisante, ils conduisent à questionner le caractère écologique de phases de familiarisation où l'on présente à l'enfant un pseudo-nom précédé de 4 consonnes de liaison /n/, /t/, /z/ et / / / (mon èque, petit èque, ces èque, dernier èque).

Malgré cette remarque, les travaux initiaux de Babineau et Shi (2011, 2013) incitent à prolonger les recherches dans la direction qu'ils ont impulsée et qui soulève la question - centrale sur le plan théorique de la relation entre formes perçues et instances mémorisées. Est-ce que l'enfant a besoin d'être confronté à de nombreuses occurrences d'une variante d'un nom particulier pour la mémoriser dans un fragment (chunk) incluant le déterminant qui l'accompagne le plus fréquemment et pouvoir ensuite la segmenter? Ou suffit-il qu'il rencontre un nombre restreint d'occurrences qu'il segmente à la volée en s'appuyant 
éventuellement sur les schémas productifs? Pour l'instant, cette question n'est pas tranchée; des pistes viendront assurément de la confrontation de méthodologies différentes.

\section{RÉFÉRENCES BIBLIOGRAPHIQUES}

Andersen, Henning (dir.). (1986). Sandhi Phenomenon in the Languages of Europe. Berlin, New York, Amsterdam : Mouton de Gruyter.

Babineau, Mireille \& Shi, Rushen. (2011). Processing of French Liaisons in Toddlers. Dans BUCLD 35: Proceedings of the 35th Annual Boston University Conference on Language Development (p. 25-37). Boston, MA : Cascadilla Press.

Babineau, Mireille \& Shi, Rushen. (2013). Acoustical Cues versus TopDown Bias in Infants' Parsing. The Journal of the Acoustical Society of America, 133(5), 3338.

Babineau, Mireille \& Shi, Rushen. (2014). Input Distribution, Linguistic Knowledge and Children's Interpretation of Word Boundaries: The Case of Liaison. Communication présentée au symposium du «19th Biennial International Conference on Infant Studies », Berlin, Allemagne.

Barbu, Stéphanie, Nardy, Aurélie, Chevrot, Jean-Pierre \& Juhel, Jacques. (2013). Language Evaluation and Use during Early Childhood: Adhesion to Social Norms or Integration of Environmental Regularities? Linguistics, 51(2), 381-411.

Berko, Jean. (1958). The Child's Learning of English Morphology. Word, 14, 150-177.

Buerkin-Salgado, Angelica, Culbertson, Jennifer, Legendre, Géraldine \& NAzzI, Thierry. (2014, juillet). The Early Acquisition of Liaison. Communication présentée au symposium du «19th Biennial International Conference on Infant Studies», Berlin, Germany.

B ybee, Joan. (2003). Phonology and Language Use. Cambridge : Cambridge University Press.

Chevrot, Jean-Pierre \& Fayol, Michel. (2000). L'acquisition de la liaison : enjeux théoriques, premiers résultats, perspectives. Lidil, 22, 11-30.

Chevrot, Jean-Pierre, FAyol, Michel \& LAKs, Bernard. (2005). La liaison : de la phonologie à la cognition. Langages, 158, 3-7.

Chevrot, Jean-Pierre, Chabanal, Damien \& Dugua, Céline. (2007). Pour un modèle de l'acquisition des liaisons basé sur l'usage : trois études de cas. Journal of French Language Studies, 17, 103-128.

Chevrot, Jean-Pierre, Dugua, Céline \& Fayol, Michel. (2009). Liaison Acquisition, Word Segmentation and Construction in French: A Usage Based Account. Journal of Child Language, 36(3), 557-596. 
Chevrot, Jean-Pierre, Nardy, Aurélie \& Barbu, Stéphanie. (2011). Developmental Dynamics of SES-Related Differences in Children's Production of Obligatory and Variable Phonological Alternations. Language Sciences, 33(1), 180-191.

Chevrot, Jean-Pierre, Dugua, Céline, Harnois-Delpiano, Mylène, SicCARDI, Anne \& SPInelli, Elsa. (2013). Liaison Acquisition, Debates, Critical Issues, Future Research. Language Sciences, 39, 83-94.

Chevrot, Jean-Pierre. (2013, novembre). Liaison Acquisition and Word Formation in French: A Specific Influence of Phonotactics? Communication présentée au «2nd International Workshop on Phonotactics and Phonotactic Modeling», Pise, Italie.

Core, Cynthia. (2012). Assessing Phonological Knowledge. Dans E. Hoff (dir.), Research Methods in Child Language. A Practical Guide (p. 7999). Malden : Blackwell Publishing.

Côté, Marie-Hélène. (2005). Le statut lexical des consonnes de liaison. Langages, 158, 66-78.

Croft, William \& Cruse, D. Alan. (2004). Cognitive Linguistics. Cambridge : Cambridge University Press.

De Jong, Daan. (1994). La sociophonologie de la liaison orléanaise. Dans C. Lyche (dir.), French Generative Phonology: Retrospective and Perspectives (p. 95-129). Salford : ESRI.

Delattre, Pierre. (1966). Studies in French and Comparative Phonetics: Selected Papers in French and English. The Hague, Londres, Paris : Mouton \& Co.

Diessel, Holger. (2004). The Acquisition of Complex Sentences. Cambridge : Cambridge University Press.

Dugua, Céline. (2006). Liaison, segmentation lexicale et schémas syntaxiques entre 2 et 6 ans : un modèle développemental basé sur l'usage (Thèse de doctorat). Université Stendhal-Grenoble 3, Grenoble.

Dugua, Céline \& Spinelli, Elsa. (2009). La liaison : effets de la fréquence et du rapport à l'écrit sur son acquisition et son usage. Cahier de l'infantile, 17-41.

Dugua, Céline, Spinelli, Elsa, Chevrot, Jean-Pierre \& Fayol, Michel. (2009). Usage-Based Account of the Acquisition of Liaison: Evidence from Sensitivity to the Singular/Plural Orientation of Nouns. Journal of Experimental Child Psychology, 102(3), 342-350.

Durand, Jacques \& Lyche, Chantal. (2008). French Liaison in the Light of Corpus Data. Journal of French Language Studies, 18(1), 33-66.

EnCREvé, Pierre. (1983). La liaison sans enchainement. Actes de la recherche en sciences sociales, 46, 39-46.

Fillmore, Charles J., Kaye, Paul \& O’connor, Mary Catherine. (1988). Regularity and Idioaticity in Grammatical Constructions: The Case of Let Alone. Language, 64(3), 501-538. 
Gallot, Sophie, Spinelli, Elsa, Chevrot, Jean-Pierre \& Dugua, Céline. (2009). Le rattachement lexical de la liaison : une expérience d'amorçage chez des enfants pré-lecteurs. Psychologie française, 54, 355-361.

Goldberg, Adèle E. (1995). A Construction Grammar Approach to Argument Structure. Chicago et Londres : The University of Chicago Press.

GoldBerg, Adèle E. (2003). Constructions: A New Theoretical Approach to Language. Trends in Cognitive Sciences, 7, 219-224.

Goyet, Louise, de Schonen, Scania \& NAzzi, Thierry. (2010). Words and Syllables in Fluent Speech Segmentation by French-Learning Infants: An ERP Study. Brain Research, 1332, 75-89.

GrégoIre, Antoine. (1947). L'apprentissage du langage II - La troisième année et les années suivantes. Genève : E. Droz.

JAnssen, Niels \& Barber, Horacio A. (2012). Phrase Frequency Effects in Language Production. PLoS ONE, 7(3), e33202. <http://dx.doi.org/ 10.1371/journal.pone.0033202>.

HöHLe, Barbara \& Weissenborn, Jürgen. (2000). Lauter Laute? Lautsegmenteund Silben in der Sprachperzeption und im Spracherwerb. Dans R. Thieroff, M. Tamrat, N. Fuhrhop \& O. Teuber (dir.), Deutsche Grammatik in Theorie und Praxis (p. 1-11). Tübingen : Niemeyer.

Kemmer, Suzanne \& Barlow, Michael. (2000). Introduction: A UsageBased Conception of Language. Dans M. Barlow \& S. Kemmer (dir.), Usage-Based Models of Language Use (p. VII-XXVIII). Stanford, Californie : CSLI Publications.

LAKs, Bernard. (2005). La liaison et l'illusion. Langages, 158, 101-126.

LANGACKer, Ronald W. (1987). Foundations of Cognitive Grammar. Stanford, Californie : Stanford University Press.

Langacker, Ronald W. (2000). A Dynamic Usage-Based Model. Dans M. Barlow \& S. Kemmer (dir.), Usage-Based Models of Language Use (p. 1-63). Stanford, Californie : CSLI Publications.

Liégeois, Loïc, Chabanal, Damien \& Chanier, Thierry. (2011, juillet). La liaison en discours adressé à l'enfant, spécificités et impacts sur l'acquisition. Communication présentée au colloque du Réseau français de phonologie, Tours, France.

Mattys, Sven L. \& JusczyK, Peter W. (2001). Do Infants Segment Words or Recurring Continuous Patterns? Journal of Experimental Psychology: Human Perception and Performance, 27, 644-655.

Morgenstern, Aliya \& Parisse, Christophe. (2012). The Paris Corpus. French Language Studies, 22(1), 7-12.

Nardy, Aurélie. (2008). Acquisition des variables sociolinguistiques entre 2 et 6 ans : facteurs sociologiques et influences des interactions au sein du réseau social (Thèse de doctorat). Université Stendhal-Grenoble 3, Grenoble. 
NARdy, Aurélie \& Dugua, Céline. (2011). Le rôle de l'usage sur le développement des constructions nominales chez les enfants pré-lecteurs. Travaux de linguistique, 162, 129-148.

Piaget, Jean. (1936). La naissance de l'intelligence chez l'enfant. Paris : Delachaux et Niestlé.

Pine, Julian M. \& Lieven, Elena. (1993). Reanalysing Rote-Learned Phrases: Individual Differences in the Transition to Multi Word Speech. Journal of Child Language, 20(3), 551-571.

Pine, Julian M. \& Lieven, Elena. (1997). Slot and Frame Patterns in the Development of the Determiner Category. Applied Psycholinguistics, 18(2), 123-138.

Pine, Julian M., Freudenthal Daniel, Krajewski Grzegorz \& Gobet Fernand. (2013). Do Young Children Have Adult-Like Syntactic Categories? Zipf's Law and the Case of the Determiner. Cognition, 127, 345-360.

Shi, Rushen, Marquis, Alexandra \& Gauthier, Bruno. (2006). Segmentation and Representation of Function Words in Preverbal FrenchLearning Infants. Dans D. Bamman, T. Magnitskaia \& C. Zaller (dir.), BUCLD 30: Proceedings of the 30th Annual Boston University Conference on Language Development (vol. 2, p. 549-560). Boston, MA : Cascadilla Press.

Shi, Rushen \& Lepage, Mélanie (2008). The Effect of Functional Morphemes on Word Segmentation in Preverbal Infants. Developmental Science, 11(3), 407-413.

Tomasello, Michael. (2003). Constructing a Language: A Usage-Based Theory of Language Acquisition. Cambridge, MA : Harvard University Press.

Wauquier-Gravelines, Sophie \& Braud, Virginie. (2005). Proto-déterminant et acquisition de la liaison obligatoire en français. Langages, 158, 53-65.

WAUquier, Sophie. (2009). Acquisition de la liaison en L1 et L2 : stratégies phonologiques ou lexicales? Aile... Lia, 2, 93-130. 